\title{
PROGRESS REPORT ON THE HERSTMONCEUX PROGAMME OF MEASUREMENT OF PROPER MOTIONS IN THE NORTHERN SELECTED AREAS
}

\author{
C. A. MURRAY \\ Royal Greenwich Observatory, Herstmonceux, England
}

\begin{abstract}
First results of the Herstmonceux programme of redetermination of proper motions in the Selected Areas are presented. Plate material includes the old Radcliffe plates, old plates taken at Greenwich and modern plates taken on the 26-in. refractor at Herstmonceux. The plates are being measured on GALAXY and reduced according to the plate-overlap technique.
\end{abstract}

\section{Introduction}

Proper motions in the northern selected areas were measured for stars down to about $m_{p g}=15$, during the 1930's at both the Radcliffe (Oxford) (Knox-Shaw, 1934) and the Pulkova observatories (Deutsch, 1940). Extensive use of this material has been made for various statistical studies, of stellar kinematics (e.g. Hins and Blaauw, 1948) and of mean parallaxes (e.g. Binnendijk, 1943). However, both sets of data were based on rather short time bases, with a result that the optimum standard errors of individual proper motions are of the order \pm ".006, and are larger for faint stars. As many of these faint stars will be at distances of between 500 and $1000 \mathrm{pc}$, the accidental errors are quite comparable with the likely velocity dispersions and hence will mask the true characteristics of the velocity distribution.

It was pointed out at IAU Symp. 1 on 'Coordination of Galactic Research' (ed. by Blaauw, 1953), that a repetition of the original plates would produce proper motions with greatly increased weight, which would make possible a much more refined study of velocity distributions of distant objects in various parts of the sky. It was also remarked that such a project would entail a large amount of labour and suggestions were then made for limiting the programme to low latitude fields.

However, with modern automatic measuring machines such as GALAXY, now available, it is possible to undertake the whole project, and indeed, the limitation of the areas measured in the original catalogues, according to galactic latitude is no longer necessary. A complete re-observation and measurement of the Radcliffe material is being undertaken at Herstmonceux, in which the full usable area of the $16 \mathrm{~cm} \times 16 \mathrm{~cm}$ plates (scale $\sim 30^{\prime \prime} \mathrm{mm}^{-1}$ ) will be measured.

Most of the original Radcliffe plates have survived and are on loan to the Royal Greenwich Observatory through the courtesy of the Director of the University of London Observatory where the original Radcliffe 24-in. refractor is now situated. A few modern plates have been taken at London with this telescope, but in the main, the repetition has been carried out at Herstmonceux with the 26-in. refractor. 
In addition, plates on some areas were taken at Greenwich for photometric use, during the period 1910-20, and these have also been repeated. First indications are, however, that the original plates in this series have considerably less weight than the Radcliffe plates.

An important feature of the present programme is the measurement of $B$ and $V$ magnitudes as far as possible for all the stars measured for proper motion. Photometric plates for this purpose are also being obtained with the 26 -in. refractor, but there is no plan at present for systematic photoelectric observations. A number of standards down to about $V=12$ in several areas have been observed over the past few years by various RGO staff members (Epps, 1972; Penston, 1973).

As reported previously (Murray and Clube, 1970), the initial plan is to measure and study the seven areas (SA, 51, 54, 57, 71, 82, 94, 107) for which Purgathofer (1969) has published photoelectric sequences.

\section{Observational Programme}

Generally the Radcliffe plates on each field consist of a pair of plates between 1909 and 1919 and a pair between 1920 and 1933. Some plates however were taken on the Kapteyn plan in which first epoch plates were stored undeveloped, and re-exposed at the second epoch thus giving two epochs on the same plate. There are thus about 450 plate epochs on rather fewer plates. About $95 \%$ of these already have been reobserved at Herstmonceux.

The Greenwich photometric series contains rather less than 100 plates on 76 areas, with a heavy concentration of plates in the $+15^{\circ}$ zone; the repetition of these is virtually complete.

The programme of new photometric $B V$ plates is under way and to date, at least two plates in each colour have been taken on 24 areas.

\section{Outline of Measurement and Reduction Procedures}

Measurement of the plates is now in progress on the GALAXY machine at Herstmonceux.

A description of the original GALAXY at Royal Observatory Edinburgh, and its operation, have been given elsewhere (Walker, 1971; Pratt, 1971).

In the search mode, the time taken to scan a full $16 \mathrm{~cm} \times 16 \mathrm{~cm}$ plate with the $32 \mu$ scanning spot is about $6 \mathrm{~h}$; therefore, in order to reduce the amount of searching in fields on which a number of plates have to be measured, computer programmes have been developed at RGO for producing search co-ordinates appropriate to each plate from data derived from searches on a few plates. The current procedure is to search two early plates and two modern plates, and to calculate an approximate search ephemeris for each star; in this way we hope to ensure that stars with large proper motion are not inadvertently missed, as they might well be if searches were carried out at one epoch only. The efficiency of the search mode is very dependent on plate 
background density, and some difficulty is being experienced with many of the old Radcliffe plates which have rather dense and variable fog levels.

All the astrometric plates are measured twice, in two opposite orientations, to eliminate magnitude equation in the measured coordinates. With this procedure we have found that the repeatability of measurement on a single plate is consistent to better than $\pm 1 \mu$ over at least nine magnitudes.

After measurement of all plates on a field, the data are processed by a series of computer programmes in which measurements on all exposures (including multiple exposures on one plate) are transformed orthogonally to a common arbitrary coordinate system and sorted, such that all the measurements for each star are collected together, and the complete set of data is output on magnetic tape for subsequent reduction.

The reduction is carried out in four main stages:

(i) Combination of the measurements made in the two orientations.

(ii) Photometric reduction using calibration from photoelectric standards.

(iii) Transformation of all measurements to standard coordinates on a common tangent plane (including correction for refraction and aberration) through AGK3 stars, and classical solution for position and proper motion components for each star.

(iv) Final reduction of all plates on a field simultaneously by the plate overlap method.

Full details of the measurement and reduction procedures will be published in due course.

\section{Preliminary Results}

The computer programmes for the reduction have been developed using measurements made on 18 plates of SA54 on the GALAXY machine at Royal Observatory Edinburgh. In all, there is a total of 66 sets of measurements on 32 exposures, four of which were on modern photometric plates with single exposures and the remainder were plates ranging in epoch from 1913 to 1970, all with double exposures.

The classical solution for position and proper motion in stage (iii) of the reduction yields standard errors of unit weight for each star in each coordinate, from the residuals. In the present tests, each exposure on a double exposure plate was treated as an independent observation, and all images were given unit weight except those on the old Greenwich photometric plates which were given weight 0.1 . In the $X$ coordinates the s.e. of unit weight for stars $8<B \leqslant 14$ is remarkably constant with a median near \pm 0 " 10 and individual values ranging from \pm 0 ".03 to 0 ".26. Since the weight of the proper motion of the majority of stars is at least $10^{4}$, this represents a formal s.e. of a proper motion component of about \pm 0 ". 001 . However, there is some evidence of correlation between the residuals of the two exposures on the same plate which may increase this value somewhat. In the current sample of data the $Y$-measurements do not appear to be as good as those in $X$; it is not clear at present whether this is in the plates themselves or in the measuring procedure. These plates were not measured in two opposite orientations as the presence of a magnitude equation in 
the GALAXY measurements was not then suspected. In both coordinates, the accuracy decreases sharply between $B=14$ and 15 reaching a mean s.e. in $X$ at $B=16$ of about \pm 0 . 35 . Nevertheless this still means that the proper motions of these faint stars have formal standard errors of only about \pm ".003.

High weight proper motions (wt. $>5000$ ) have been obtained for 230 stars in the field compared with 144 stars in the Radcliffe catalogue. These include several fainter stars over the whole field as well as stars outside the area measured at Radcliffe. The proper motions are virtually complete to $B=16$.

Only preliminary tests on the overlap reduction have so far been carried out, due to limitation of computer power, but a limited solution using plate constants and simple magnitude equation has been achieved for a small subset (10 exposures) of the total available data. In this solution the usual constraints on the relative proper motion system (i.e. no translation, shear or rotation) were applied, and also the magnitude equations were constrained so that their time weighted average was zero.

Investigations into a suitable weighting system for the final overlap solution programme are under way. It is intended that this should take account of image size (which is automatically recorded in measurement on GALAXY) as well as plate quality. Information for this is being obtained from comparison of the two exposures on each plate.

\section{Acknowledgements}

The computer programmes for this work and for general use with GALAXY have been written by a number of members of the staff of the Astrometry Department. Mr W. Nicholson, with the assistance of Mrs P. Fosbury, has developed the general purpose data processing and sorting routines for GALAXY; Mrs S. B. Tritton has written programmes for generating search data, prior to measurement and has also developed the photometric reduction programmes; the present author has been responsible for stages (i) and (iii) of the reduction outlined above, and the programme for the final plate overlap is being developed by Dr B. F. Jones.

The daily operation of the GALAXY machine is carried out by Mr E. D. Clements and $\mathrm{Mr}$ B. S. Carter. Mr Clements also organised the observations on the 26-in. refractor.

The efficient use of GALAXY requires considerable use of the computer and I am grateful to the staff of the Computer section of HM Nautical Almanac Office for their assistance in this and all computing work associated with GALAXY.

I am also grateful to Dr Neil Pratt of Royal Observatory Edinburgh for his help with the measurement of the SA 54 plates on the Edinburgh GALAXY, and to $\mathrm{Mr}$ E. W. Foster and Mr D. J. Carnochen for taking plates on the 24-in. refractor at University of London Observatory.

\section{References}

Binnendijk, L.: 1943, Bull. Astron. Inst. Neth. X, 9.

Blaauw, A. (ed.): 1955, in 'Co-ordination of Galactic Research', IAU Symp. 1, 35. 
Deutsch, A. N.: 1940, Pulkovo Publ. II, LV.

Hins, C. H. and Blaauw, A.: 1948, Bull. Astron. Inst. Neth. X, 365.

Epps, E. A.: 1972, R. Obs. Bull., No. 176.

Knox-Shaw, H. and Scott Barrett, H. G., 1934, The Radcliffe Catalogue of Proper Motions (O.U.P.).

Murray, C. A. and Clube, S. V. M.: 1970, in W. J. Luyten (ed.), 'Proper Motions', IAU Colloq. 7, 143.

Penston, M. J.: 1973, Monthly Notices Roy. Astron. Soc. 164, 121.

Pratt, N. M.: 1971, Publ. Roy. Obs. Edinburgh 8, 109.

Purgathofer, A. Th.: 1969, Lowell Obs. Bull., No. 147.

Walker, G. S.: 1971, Publ. Roy. Obs. Edinburgh 8, 109. 